\title{
LMI-Based Stability Criterion for Impulsive CGNNs via Fixed Point Theory
}

\author{
Xiongrui Wang, ${ }^{1,2}$ Ruofeng Rao, ${ }^{2}$ and Shouming Zhong ${ }^{3}$ \\ ${ }^{1}$ Department of Mathematics, Yibin University, Yibin 644007, China \\ ${ }^{2}$ Department of Mathematics, Chengdu Normal University, Chengdu 611130, China \\ ${ }^{3}$ School of Science Mathematics, University of Electronic Science and Technology of China, Chengdu 610054, China
}

Correspondence should be addressed to Ruofeng Rao; ruofengrao@163.com

Received 1 July 2015; Revised 30 September 2015; Accepted 5 October 2015

Academic Editor: Xinggang Yan

Copyright (C) 2015 Xiongrui Wang et al. This is an open access article distributed under the Creative Commons Attribution License, which permits unrestricted use, distribution, and reproduction in any medium, provided the original work is properly cited.

\begin{abstract}
Linear matrices inequalities (LMIs) method and the contraction mapping theorem were employed to prove the existence of globally exponentially stable trivial solution for impulsive Cohen-Grossberg neural networks (CGNNs). It is worth mentioning that it is the first time to use the contraction mapping theorem to prove the stability for CGNNs while only the Leray-Schauder fixed point theorem was applied in previous related literature. An example is given to illustrate the effectiveness of the proposed methods due to the large allowable variation range of impulse.
\end{abstract}

\section{Introduction and Preliminaries}

Dynamics of Cohen-Grossberg neural networks (CGNNs) has been extensively investigated due to its immense potentials of application perspective in various areas such as pattern recognition, parallel computing, associate memory, combinational optimization, and signal and image processing. However, these successful applications always depend on the stability of the equilibrium solution for CGNNs. All the time, the method of Lyapunov theory has usually been employed to solve the stability problem of dynamical systems [1-11]. In studying the stability of neural networks, LyapunovKrasovskii functional method can always be combined with other methods in a perfect way, such as the linear matrix inequality (LMI) optimization approach, $M$-Matrix theory, and nonsmooth analysis technique (see, e.g., [6-8]). Of course, as one of stability analysis methods, the Lyapunov method has its limitations. In fact, a stability criterion is regarded as an effective and efficient method for impulsive neural networks if a larger variation range of impulse is allowable (see, e.g., [12, Remark 11]). Thereby, using the methods different from Lyapunov direct method may obtain a more efficient stability criterion for impulsive systems. Indeed, fixed point theories have always been considered by many authors. Burton [13, 14], Rao and Pu [15], Jung [16], Luo [17], Zhang [18], and Wu et al. [19] studied the stability by using the fixed point theory which solved the difficulties encountered in the study of stability by means of Lyapunov's direct method. Contraction mapping theorem was the usual method to study the stability of neural networks, except CGNNs. Owing to some difficulties, only the Leray-Schauder fixed point theorem was considered in investigating the stability of CGNNs [20, 21]. In this paper, contraction mapping theorem is applied to the stability analysis for CGNNs. We wish that our newly obtained stability criterion will allow a larger variation range of impulses against a series of previously related literatures. This is the main purpose of this paper.

Consider the following CGNNs:

$$
\begin{array}{r}
\frac{d x_{j}(t)}{d t}=-a_{j}\left(x_{j}(t)\right)\left\{b_{j}\left(x_{j}(t)\right)\right. \\
\left.-\sum_{k=1}^{n}\left[c_{j k} f_{k}\left(x_{k}(t)\right)+d_{j k} g_{k}\left(x_{k}\left(t-\tau_{j}(t)\right)\right)\right]\right\}, \\
t \geqslant 0, t \neq t_{i},
\end{array}
$$




$$
\begin{array}{ll}
\Delta x_{j}\left(t_{i}\right)=x_{j}\left(t_{i}^{+}\right)-x_{j}\left(t_{i}\right)=\rho_{j i}\left(x_{j}\left(t_{i}\right)\right), & \\
& i=1,2, \ldots, \\
x_{j}(\theta)=\phi_{j}(\theta), \quad-\tau \leqslant \theta \leqslant 0, &
\end{array}
$$

where $j \in \mathcal{N} \triangleq\{1,2, \ldots, n\} . a_{j}\left(x_{j}(t)\right)$ and $b_{j}\left(x_{j}(t)\right)$ represent an amplification function at time $t$ and an appropriately behaved function at time $t . f_{k}(\cdot)$ and $g_{k}(\cdot)$ are the activation functions of the neurons, and $c_{j k}$ and $d_{j k}$ are connection parameters. The time-varying delays $\tau_{j}(t) \in[0, \tau]$. The impulsive moments $t_{i}(i=1,2, \ldots)$ satisfy $0=t_{0}<t_{1}<t_{2}<$ $\cdots$, and $\lim _{i \rightarrow+\infty} t_{i}=+\infty \cdot x_{j}\left(t_{i}^{+}\right)$and $x_{j}\left(t_{i}^{-}\right)$stand for the right-hand and left-hand limit of $x_{j}(t)$ at time $t_{i}$, respectively. $\rho_{j i}\left(x_{j}\left(t_{i}\right)\right)$ means the abrupt change of $x_{j}(t)$ at the impulsive moment $t_{i}$ and $\rho_{j i}(\cdot) \in C[R, R]$.

Throughout this paper, we assume that $b_{j}(0)=f_{j}(0)=$ $g_{j}(0)=\rho_{j i}(0)=0$ for $j \in \mathcal{N}$ and $i=1,2, \ldots$ Denote by $x(t) \triangleq$ $x(t ; \theta, \phi)=\left(x_{1}\left(t ; \theta, \phi_{1}\right), \ldots, x_{n}\left(t ; \theta, \phi_{n}\right)\right)^{T} \in R^{n}$ the solution for system (1) with the initial condition $x_{j}(\theta)=\phi_{j}(\theta),-\tau \leqslant$ $\theta \leqslant 0, j \in \mathcal{N}$, where $\phi(\theta)=\left(\phi_{1}(\theta), \ldots, \phi_{n}(\theta)\right)^{T} \in R^{n}$ and $\phi_{j}(\theta) \in C[[-\tau, 0], R]$. The solution $x(t) \triangleq x(t ; \theta, \phi) \in R^{n}$ of system (1) is, for the time variable $t$, a continuous vectorvalued function.

Throughout this paper, we assume the following.

(H1) For any $j \in \mathcal{N}$, there exist constants $\bar{F}_{j}>0, \bar{G}_{j}>0$, $F_{j}>0$, and $G_{j}>0$ such that

$$
\begin{gathered}
\left|f_{j}(r)\right| \leqslant \bar{F}_{j}, \\
\left|g_{j}(r)\right| \leqslant \bar{G}_{j}, \\
\left|f_{j}(r)-f_{j}(s)\right| \leqslant F_{j}|r-s|, \\
\left|g_{j}(r)-g_{j}(s)\right| \leqslant G_{j}|r-s|,
\end{gathered}
$$

$\forall r, s \in R$.

(H2) For any $j \in \mathcal{N}, a_{j}(\cdot)$ is differentiable, and there exists a constant $\widetilde{a}_{j}$ such that

$$
\begin{aligned}
0 & <a_{j}(r) \leqslant \bar{a}_{j}, \\
\left|a_{j}^{\prime}(r)\right| & \leqslant \widetilde{a}_{j},
\end{aligned}
$$

$$
r \in R \text {. }
$$

(H3) There exist nonnegative constants $h_{j i}$ such that

$$
\begin{aligned}
\left|\rho_{j i}(r)-\rho_{j i}(s)\right| \leqslant h_{j i}|r-s|, & \\
& \forall r, s \in R, j \in \mathcal{N}, i=1,2, \ldots
\end{aligned}
$$

(H4) For $j \in \mathcal{N}$, there exist $\Upsilon_{j}(t)$ and a constant $\gamma_{j}>0$ such that

$$
\begin{gathered}
\Upsilon_{j}(t) \geqslant \gamma_{j}, \quad \forall t \geqslant 0, \\
a_{j}(r) b_{j}(r)=\Upsilon_{j}(t) r, \quad r \in R .
\end{gathered}
$$

Remark 1. $a_{j}(\cdot)$ represents an amplification function; $b_{j}(\cdot)$ is an appropriate behavior function. There exist a lot of functions satisfying the above assumptions. For example, let $j \in \mathcal{N} \triangleq\{1,2\}, b_{1}\left(x_{1}(t)\right)=2 x_{1}(t), b_{2}\left(x_{2}(t)\right)=2 x_{2}(t)$, $a_{1}\left(x_{1}(t)\right)=a_{2}\left(x_{2}(t)\right)=2+\cos t$, and then $a_{j}(r) b_{j}(r)=$ $2(2+\cos t) r=\Upsilon_{j}(t) r$; hence $\Upsilon_{j}(t)=2(2+\cos t)$. Now we let $\gamma_{j}=2$, and obviously $\Upsilon_{j}(t) \geqslant \gamma_{j}$.

From the above assumptions, it is obvious that the null solution is a trivial solution for system (1).

Definition 2. System (1) is said to be globally exponentially stable if for any initial condition $\phi(\theta) \in C\left[[-\tau, 0], R^{n}\right]$ there exist positive constants $\lambda$ and $\mu$ such that

$$
\|x(t ; \theta, \phi)\| \leqslant \lambda e^{-\mu t}, \quad \forall t \geqslant 0 .
$$

Lemma 3 (see [22]). Let $\pi$ be a contraction operator on a complete metric space $X$; then there exists a unique point $\xi \in X$ for which $\pi(\xi)=\xi$.

\section{Main Result}

Before giving the main result, we may firstly definite some matrices as follows:

$$
\begin{aligned}
& C=\left(\left|c_{j k}\right|\right)_{n \times n}, \\
& D=\left(\left|d_{j k}\right|\right)_{n \times n}, \\
& \widetilde{A}=\operatorname{diag}\left(\tilde{a}_{1}, \ldots, \tilde{a}_{n}\right), \\
& \bar{A}=\operatorname{diag}\left(\bar{a}_{1}, \ldots, \bar{a}_{n}\right), \\
& \bar{F}=\operatorname{diag}\left(\bar{F}_{1}, \ldots, \bar{F}_{n}\right), \\
& \bar{G}=\operatorname{diag}\left(\bar{G}_{1}, \ldots, \bar{G}_{n}\right), \\
& F=\operatorname{diag}\left(F_{1}, \ldots, F_{n}\right), \\
& G=\operatorname{diag}\left(G_{1}, \ldots, G_{n}\right), \\
& \Gamma=\operatorname{diag}\left(\gamma_{1}, \ldots, \gamma_{n}\right) .
\end{aligned}
$$

Assume, in addition, there exists the constant $h_{j}$ with $h_{j i} \leqslant$ $h_{j} \mu$ for each $j \in \mathcal{N}$, and $\mu$ is a positive constant, satisfying $\mu \leqslant \inf _{i=1,2, \ldots}\left\{t_{i}-t_{i-1}\right\}$. Denote $H=\operatorname{diag}\left(h_{1}, \ldots, h_{n}\right)$.

Theorem 4. If there exists a positive constant $\alpha<1$ such that the following LMI condition holds:

$$
C \bar{F} \widetilde{A}+D \bar{G} \widetilde{A}+H+\mu \Gamma+C F \bar{A}+D G \bar{A} \leqslant \alpha \Gamma \bar{A},
$$

then system (1) is globally exponentially stable. 
Proof. Our proof is based on the contraction mapping principle (Lemma 3), which may be divided into four steps.

Step 1. We may set up a space frame.

Let $\Theta=\Theta_{1} \times \Theta_{2} \times \cdots \times \Theta_{n}$, and let $\Theta_{j}(j \in \mathcal{N})$ be the space consisting of functions $q_{j}(t):[-\tau, \infty) \rightarrow R$ such that, for any $j \in \mathcal{N}$,

(a) $q_{j}(t)$ is continuous on $t \in[0, \infty)$ with $t \neq t_{i}(i=$ $1,2, \ldots)$;

(b) $q_{j}(\theta)=\phi_{j}(\theta),-\tau \leqslant \theta \leqslant 0$;

(c) $e^{\beta t} q_{j}(t) \rightarrow 0$ as $t \rightarrow+\infty$, where $\beta>0$ is the constant with $\beta<\min \left\{\liminf _{t \rightarrow+\infty}\left(\int_{0}^{t} \Upsilon_{j}(s) d s / t\right), \gamma_{j}\right\}$;

(d) both $\lim _{t \rightarrow t_{i}^{-}} q_{j}(t)$ and $\lim _{t \rightarrow t_{i}^{+}} q_{j}(t)$ exist; besides, $\lim _{t \rightarrow t_{i}^{-}} q_{j}(t)=q_{j}\left(t_{i}\right)$ for $i=1,2, \ldots$.

Moreover, $\Theta$ is a complete metric space if it is equipped with a metric defined by

$$
\operatorname{dist}(q, \breve{q})=\sum_{j=1}^{n}\left(\sup _{t \geqslant-\tau}\left|q_{j}(t)-\breve{q}_{j}(t)\right|\right) \text {, }
$$

where $q=q(t)=\left(q_{1}(t), q_{2}(t), \ldots, q_{n}(t)\right)^{T} \in \Theta$ and $\breve{q}=\breve{q}(t)=$ $\left(\breve{q}_{1}(t), \breve{q}_{2}(t), \ldots, \breve{q}_{n}(t)\right)^{T} \in \Theta$.

Remark 5. It is followed by $\beta<\liminf _{t \rightarrow+\infty}\left(\int_{0}^{t} \Upsilon_{j}(s) d s / t\right)$ that

$$
\begin{aligned}
-\infty & \leqslant \liminf _{t \rightarrow+\infty}\left(\beta-\frac{\int_{0}^{t} \Upsilon_{j}(s) d s}{t}\right) t \\
& \leqslant \limsup _{t \rightarrow+\infty}\left(\beta-\frac{\int_{0}^{t} \Upsilon_{j}(s) d s}{t}\right) t \\
& \leqslant\left(\beta-\liminf _{t \rightarrow+\infty} \frac{\int_{0}^{t} \Upsilon_{j}(s) d s}{t}\right) \limsup _{t \rightarrow+\infty} t \leqslant-\infty,
\end{aligned}
$$

and hence

$$
\lim _{t \rightarrow+\infty} e^{\left(\beta t-\int_{0}^{t} \Upsilon_{j}(s) d s\right)}=0
$$

Step 2. Frame a mapping on the space $\Theta$.
Multiplying both sides of system (1) with $e^{\int_{0}^{t} \Upsilon_{j}(s) d s}$ yields, for $t>0$ and $t \neq t_{i}(i=1,2, \ldots)$,

$$
\begin{aligned}
& \frac{d\left(e^{\int_{0}^{t} \Upsilon_{j}(s) d s} x_{j}(t)\right)}{d t}=\Upsilon_{j}(t) e^{\int_{0}^{t} \Upsilon_{j}(s) d s} x_{j}(t)+e^{\int_{0}^{t} \Upsilon_{j}(s) d s} \\
& \cdot \frac{d x_{j}(t)}{d t}=\Upsilon_{j}(t) e^{\int_{0}^{t} \Upsilon_{j}(s) d s} x_{j}(t) \\
& +e^{\int_{0}^{t} \Upsilon_{j}(s) d s}\left\{-a_{j}\left(x_{j}(t)\right)\left[b_{j}\left(x_{j}(t)\right)\right.\right. \\
& \left.\left.-\sum_{k=1}^{n}\left(c_{j k} f_{k}\left(x_{k}(t)\right)+d_{j k} g_{k}\left(x_{k}\left(t-\tau_{j}(t)\right)\right)\right)\right]\right\} \\
& =e^{\int_{0}^{t} \Upsilon_{j}(s) d s} a_{j}\left(x_{j}(t)\right) \sum_{k=1}^{n}\left(c_{j k} f_{k}\left(x_{k}(t)\right)\right. \\
& \left.+d_{j k} g_{k}\left(x_{k}\left(t-\tau_{j}(t)\right)\right)\right) .
\end{aligned}
$$

Let $\varepsilon>0$ be small enough. After integrating from $t_{i-1}+\varepsilon$ to $t \in\left(t_{i-1}, t_{i}\right)(i=1,2, \ldots)$, we get

$$
\begin{aligned}
& e^{\int_{0}^{t} \Upsilon_{j}(s) d s} x_{j}(t)-e^{\int_{0}^{t_{i-1}+\varepsilon} \Upsilon_{j}(s) d s} x_{j}\left(t_{i-1}+\varepsilon\right) \\
& =\int_{t_{i-1}+\varepsilon}^{t} e^{\int_{0}^{s} \Upsilon_{j}(l) d l} a_{j}\left(x_{j}(s)\right) \\
& \cdot \sum_{k=1}^{n}\left(c_{j k} f_{k}\left(x_{k}(s)\right)+d_{j k} g_{k}\left(x_{k}\left(s-\tau_{j}(s)\right)\right)\right) d s .
\end{aligned}
$$

Letting $\varepsilon \rightarrow 0$ in (13), we have

$$
\begin{array}{r}
e^{\int_{0}^{t} \Upsilon_{j}(s) d s} x_{j}(t)-e^{\int_{0}^{t_{i-1}} \Upsilon_{j}(s) d s} x_{j}\left(t_{i-1}^{+}\right) \\
=\int_{t_{i-1}}^{t} e^{\int_{0}^{s} \Upsilon_{j}(l) d l} a_{j}\left(x_{j}(s)\right) \\
\cdot \sum_{k=1}^{n}\left(c_{j k} f_{k}\left(x_{k}(s)\right)+d_{j k} g_{k}\left(x_{k}\left(s-\tau_{j}(s)\right)\right)\right) d s, \\
t \in\left(t_{i-1}, t_{i}\right)(i=1,2, \ldots) .
\end{array}
$$

Setting $t=t_{i}-\varepsilon(\varepsilon>0)$ in (14), we obtain

$$
\begin{aligned}
& e^{\int_{0}^{t_{i}-\varepsilon} \Upsilon_{j}(s) d s} x_{j}\left(t_{i}-\varepsilon\right)-e^{\int_{0}^{t_{i-1}} \Upsilon_{j}(s) d s} x_{j}\left(t_{i-1}^{+}\right) \\
& =\int_{t_{i-1}}^{t_{i}-\varepsilon} e^{\int_{0}^{s} \Upsilon_{j}(l) d l} a_{j}\left(x_{j}(s)\right) \\
& \cdot \sum_{k=1}^{n}\left(c_{j k} f_{k}\left(x_{k}(s)\right)+d_{j k} g_{k}\left(x_{k}\left(s-\tau_{j}(s)\right)\right)\right) d s,
\end{aligned}
$$


which yields by letting $\varepsilon \rightarrow 0$

$$
\begin{aligned}
e^{\int_{0}^{t_{i}}} \Upsilon_{j}(s) d s & x_{j}\left(t_{i}\right)-e^{\int_{0}^{t_{i}-1} \Upsilon_{j}(s) d s} x_{j}\left(t_{i-1}^{+}\right) \\
= & e^{\int_{0}^{t_{i}} \Upsilon_{j}(s) d s} x_{j}\left(t_{i}^{-}\right)-e^{\int_{0}^{t_{i-1}} \Upsilon_{j}(s) d s} x_{j}\left(t_{i-1}^{+}\right) \\
= & \int_{t_{i-1}}^{t_{i}} e^{\int_{0}^{s} \Upsilon_{j}(l) d l} a_{j}\left(x_{j}(s)\right) \\
& \cdot \sum_{k=1}^{n}\left(c_{j k} f_{k}\left(x_{k}(s)\right)+d_{j k} g_{k}\left(x_{k}\left(s-\tau_{j}(s)\right)\right)\right) d s .
\end{aligned}
$$

Combining (14) and (16) results in

$$
\begin{aligned}
& e^{\int_{0}^{t} Y_{j}(s) d s} x_{j}(t)-e^{\int_{0}^{t_{i-1}} Y_{j}(s) d s}\left(x_{j}\left(t_{i-1}\right)\right. \\
& \left.+\rho_{j(i-1)}\left(x_{j}\left(t_{i-1}\right)\right)\right)=e^{\int_{0}^{t} Y_{j}(s) d s} x_{j}(t) \\
& -e^{\int_{0}^{t_{i-1}} Y_{j}(s) d s} x_{j}\left(t_{i-1}^{+}\right)=\int_{t_{i-1}}^{t} e^{\int_{0}^{s} Y_{j}(l) d l} a_{j}\left(x_{j}(s)\right) \\
& \cdot \sum_{k=1}^{n}\left(c_{j k} f_{k}\left(x_{k}(s)\right)+d_{j k} g_{k}\left(x_{k}\left(s-\tau_{j}(s)\right)\right)\right) d s, \\
& t_{i-1}<t \leqslant t_{i}(i=1,2, \ldots) .
\end{aligned}
$$

Hence,

$$
\begin{aligned}
& e^{\int_{0}^{t_{i-1}} \Upsilon_{j}(s) d s} x_{j}\left(t_{i-1}\right)-e^{\int_{0}^{t_{i-2}} \Upsilon_{j}(s) d s}\left(x_{j}\left(t_{i-2}\right)\right. \\
& \left.+\rho_{j(i-2)}\left(x_{j}\left(t_{i-2}\right)\right)\right)=\int_{t_{i-2}}^{t_{i-1}} e^{s} \Upsilon_{0}^{s(l) d l} a_{j}\left(x_{j}(s)\right) \\
& \cdot \sum_{k=1}^{n}\left(c_{j k} f_{k}\left(x_{k}(s)\right)+d_{j k} g_{k}\left(x_{k}\left(s-\tau_{j}(s)\right)\right)\right) d s,
\end{aligned}
$$$$
e^{\int_{0}^{t_{2}} \Upsilon_{j}(s) d s} x_{j}\left(t_{2}\right)-e^{\int_{0}^{t_{1}} \Upsilon_{j}(s) d s}\left(x_{j}\left(t_{1}\right)+\rho_{j 1}\left(x_{j}\left(t_{1}\right)\right)\right)
$$$$
=\int_{t_{1}}^{t_{2}} e^{\int_{0}^{s} \gamma_{j}(l) d l} a_{j}\left(x_{j}(s)\right)
$$$$
\cdot \sum_{k=1}^{n}\left(c_{j k} f_{k}\left(x_{k}(s)\right)+d_{j k} g_{k}\left(x_{k}\left(s-\tau_{j}(s)\right)\right)\right) d s,
$$$$
e^{\int_{0}^{t_{1}} \gamma_{j}(s) d s} x_{j}\left(t_{1}\right)-\phi_{j}(0)=\int_{0}^{t_{1}} e^{\int_{0}^{s} \gamma_{j}(l) d l} a_{j}\left(x_{j}(s)\right)
$$$$
\cdot \sum_{k=0}^{n}\left(c_{j k} f_{k}\left(x_{k}(s)\right)+d_{j k} g_{k}\left(x_{k}\left(s-\tau_{j}(s)\right)\right)\right) d s,
$$

which produces

$$
\begin{aligned}
& x_{j}(t)=e^{-\int_{0}^{t} Y_{j}(s) d s} \phi_{j}(0) \\
& +e^{-\int_{0}^{t} Y_{j}(s) d s} \int_{0}^{t} e^{\int_{0}^{s} Y_{j}(l) d l}\left[a_{j}\left(x_{j}(s)\right)\right. \\
& \left.\cdot \sum_{k=1}^{n}\left(c_{j k} f_{k}\left(x_{k}(s)\right)+d_{j k} g_{k}\left(x_{k}\left(s-\tau_{j}(s)\right)\right)\right)\right] d s \\
& +e^{-\int_{0}^{t} Y_{j}(s) d s} \sum_{0<t_{i}<t}\left(\rho_{j i}\left(x_{j}\left(t_{i}\right)\right)\right) e^{\int_{0}^{t_{i}} Y_{j}(s) d s}, \quad t>0 .
\end{aligned}
$$

Thereby, we may define the mapping $\Phi: x(t) \rightarrow$ $\Phi(x)(t)$, where $x(t)=\left(x_{1}(t), \ldots, x_{j}(t), \ldots, x_{n}(t)\right)^{T} \in \Theta$, and $\Phi(x)(t)=\left(\Phi\left(x_{1}\right)(t), \ldots, \Phi\left(x_{j}\right)(t), \ldots, \Phi\left(x_{n}\right)(t)\right)^{T}$. In addition, for any $j \in \mathscr{N}$, the mapping $\Phi\left(x_{j}\right)(t):[-\tau, \infty) \rightarrow$ $R$ satisfies

$$
\begin{aligned}
& \Phi\left(x_{j}\right)(t)=e^{-\int_{0}^{t} \Upsilon_{j}(s) d s} \phi_{j}(0) \\
& +\int_{0}^{t} e^{-\int_{s}^{t} \Upsilon_{j}(l) d l}\left[a_{j}\left(x_{j}(s)\right)\right. \\
& \left.\quad \cdot \sum_{k=1}^{n}\left(c_{j k} f_{k}\left(x_{k}(s)\right)+d_{j k} g_{k}\left(x_{k}\left(s-\tau_{j}(s)\right)\right)\right)\right] d s \\
& +e^{-\int_{0}^{t} \Upsilon_{j}(s) d s} \sum_{0<t_{i}<t}\left(\rho_{j i}\left(x_{j}\left(t_{i}\right)\right) e^{\int_{0}^{t_{i}} \gamma_{j}(s) d s}\right), \quad t \geqslant 0,
\end{aligned}
$$

and $\Phi\left(x_{j}\right)(\theta)=\phi_{j}(\theta)$ as $-\tau \leqslant \theta \leqslant 0$.

Next, we will prove that $\Phi\left(\Theta_{j}\right) \subset \Theta_{j}, \forall j \in \mathcal{N}$.

It is obvious that conditions (a) and (b) hold in $\Phi\left(\Theta_{j}\right)$. So we only need to prove that, for any $x_{j}(t) \in \Theta_{j}$,

$$
\left|e^{\beta t} \Phi\left(x_{j}\right)\right| \longrightarrow 0, \quad t \longrightarrow+\infty .
$$

Indeed, it is obvious that

$$
\left|e^{\beta t} \Phi\left(x_{j}\right)\right| \leqslant Q_{1}+Q_{2}+Q_{3}+Q_{4},
$$

where

$$
\begin{aligned}
Q_{1} & =\left|e^{\beta t} e^{-\int_{0}^{t} Y_{j}(s) d s} \phi_{j}(0)\right|, \\
Q_{2} & =\left|e^{\beta t} \int_{0}^{t} e^{-\int_{s}^{t} \gamma_{j}(l) d l} a_{j}\left(x_{j}(s)\right) \sum_{k=1}^{n} c_{j k} f_{k}\left(x_{k}(s)\right) d s\right|, \\
Q_{3} & =\mid e^{\beta t} \int_{0}^{t} e^{-\int_{s}^{t} Y_{j}(l) d l} a_{j}\left(x_{j}(s)\right) \\
\cdot & \sum_{k=1}^{n} d_{j k} g_{k}\left(x_{k}\left(s-\tau_{j}(s)\right)\right) d s \mid \\
Q_{4} & =\left|e^{\beta t} e^{-\int_{0}^{t} Y_{j}(s) d s} \sum_{0<t_{i}<t}\left(\rho_{j i}\left(x_{j}\left(t_{i}\right)\right)\right) e^{\int_{0}^{t_{i}} \Upsilon_{j}(s) d s}\right| .
\end{aligned}
$$


It follows by $\beta<\liminf _{t \rightarrow+\infty}\left(\int_{0}^{t} \Upsilon_{j}(s) d s / t\right)$ that $e^{\beta t} e^{-\int_{0}^{t} Y_{j}(s) d s}=e^{\left(\beta-\int_{0}^{t} Y_{j}(s) d s / t\right) t} \rightarrow 0$ as $t \rightarrow+\infty$. And hence $Q_{1}=\left|e^{\beta t} e^{-\int_{0}^{t} \gamma_{j}(s) d s} \phi_{j}(0)\right| \rightarrow 0$ as $t \rightarrow \infty$.

Note that $e^{\beta t} x_{j}(t) \rightarrow 0$ if $x_{j}(t) \in \Theta_{j}, \forall j \in \mathcal{N}$. So, for any $\varepsilon>0$, there exists a positive constant $N$ such that

$$
\left|e^{\beta t} x_{j}(t)\right|<\varepsilon, \quad \forall t \in[N,+\infty) .
$$

Thereby,

$$
\begin{aligned}
\left|e^{\int_{0}^{t} \gamma_{j}(s) d s} x_{j}(t)\right| & =e^{\left(\int_{0}^{t} Y_{j}(s) d s-\beta t\right)}\left|e^{\beta t} x_{j}(t)\right| \\
& \leqslant e^{\left(\int_{0}^{t} Y_{j}(s) d s-\beta t\right)} \varepsilon, \quad \forall t \in[N,+\infty) .
\end{aligned}
$$

The continuity of $x_{j}(t)$ derives that there exists a constant $M>0$ such that

$$
\begin{aligned}
\left|e^{\beta t} x_{j}(t)\right| & <M, \quad \forall t \in[0, N], \\
\left|e^{\int_{0}^{t} Y_{j}(s) d s} x_{j}(t)\right| & =e^{\left(\int_{0}^{t} Y_{j}(s) d s-\beta t\right)}\left|e^{\beta t} x_{j}(t)\right| \\
& \leqslant M \max _{t \in[0, N]}\left(e^{\left(\int_{0}^{t} Y_{j}(s) d s-\beta t\right)}\right),
\end{aligned}
$$

$\forall t \in[0, N]$.

In addition, condition (H1) yields that $\left|f_{k}\left(x_{k}(s)\right)\right|=$ $\left|f_{k}\left(x_{k}(s)\right)-f_{k}(0)\right| \leqslant F_{k}\left|x_{k}(s)-0\right|$. Let $t>N$, and we can derive from (25), (27), and the change of variable formula for integrals that

$$
\begin{aligned}
& Q_{2} \leqslant e^{-\left(\int_{0}^{t} Y_{j}(s) d s-\beta t\right)}\left[\int_{0}^{N} \bar{a}_{j} \sum_{k=1}^{n}\left|c_{j k}\right|\right. \\
& \cdot F_{k}\left|e^{\int_{0}^{s} Y_{j}(l) d l} x_{k}(s)\right| d s+\int_{N}^{t} \bar{a}_{j} \sum_{k=1}^{n}\left|c_{j k}\right| \\
& \left.\cdot F_{k}\left|e^{\int_{0}^{s} Y_{j}(l) d l} x_{k}(s)\right| d s\right] \leqslant e^{-\left(\int_{0}^{t} Y_{j}(s) d s-\beta t\right)}[M \\
& \cdot \max _{t \in[0, N]}\left(e^{\left(\int_{0}^{t} Y_{j}(s) d s-\beta t\right)}\right) \int_{0}^{N} \bar{a}_{j} \sum_{k=1}^{n}\left|c_{j k}\right| F_{k} d s \\
& \left.+\bar{a}_{j} \sum_{k=1}^{n}\left|c_{j k}\right| F_{k} \varepsilon \int_{N}^{t} e^{\left(\int_{0}^{s} Y_{j}(l) d l-\beta s\right)} d s\right] \\
& \leqslant e^{-\left(\int_{0}^{t} Y_{j}(s) d s-\beta t\right)}\left[M \max _{t \in[0, N]}\left(e^{\left(\int_{0}^{t} \Upsilon_{j}(s) d s-\beta t\right)}\right)\right. \\
& \left.\cdot N \bar{a}_{j} \sum_{k=1}^{n}\left|c_{j k}\right| F_{k}\right]+\frac{\bar{a}_{j} \sum_{k=1}^{n}\left|c_{j k}\right| F_{k}}{\gamma_{j}-\beta} \varepsilon .
\end{aligned}
$$

Now we can conclude from the arbitrariness of $\varepsilon$ that $Q_{2} \rightarrow$ $0, t \rightarrow \infty$.
Similarly, condition $(\mathrm{H} 2)$ yields

$$
\begin{aligned}
Q_{3} & \leqslant e^{-\left(\int_{0}^{t} Y_{j}(s) d s-\beta t\right)} \int_{0}^{t} e^{\int_{0}^{s} Y_{j}(l) d l} \bar{a}_{j} \sum_{k=1}^{n}\left|d_{j k}\right| \\
& \cdot G_{k}\left|x_{k}\left(s-\tau_{j}(s)\right)\right| d s \\
& \leqslant e^{\beta \tau} e^{-\left(\int_{0}^{t} Y_{j}(s) d s-\beta t\right)} \bar{a}_{j} \sum_{k=1}^{n}\left|d_{j k}\right| G_{k} \\
& \cdot \int_{0}^{t} e^{\left(\int_{0}^{s} Y_{j}(l) d l-\beta s\right)}\left|e^{\beta\left(s-\tau_{j}(s)\right)} x_{k}\left(s-\tau_{j}(s)\right)\right| d s .
\end{aligned}
$$

Let $t>N+\tau$; we can get by (24)

$$
\begin{aligned}
& \int_{0}^{t} e^{\left(\int_{0}^{s} \gamma_{j}(l) d l-\beta s\right)}\left|e^{\beta\left(s-\tau_{j}(s)\right)} x_{k}\left(s-\tau_{j}(s)\right)\right| d s \\
& \quad=\int_{0}^{N+\tau} e^{\left(\int_{0}^{s} \gamma_{j}(l) d l-\beta s\right)}\left|e^{\beta\left(s-\tau_{j}(s)\right)} x_{k}\left(s-\tau_{j}(s)\right)\right| d s \\
& \quad+\int_{N+\tau}^{t} e^{\left(\int_{0}^{s} \gamma_{j}(l) d l-\beta s\right)}\left|e^{\beta\left(s-\tau_{j}(s)\right)} x_{k}\left(s-\tau_{j}(s)\right)\right| d s \\
& \quad \leqslant \sup _{s \in[0, N+\tau]}\left|e^{\beta\left(s-\tau_{j}(s)\right)} x_{k}\left(s-\tau_{j}(s)\right)\right| \\
& \cdot \int_{0}^{N+\tau} e^{\left(\int_{0}^{s} Y_{j}(l) d l-\beta s\right)} d s+\int_{N+\tau}^{t} e^{\left(\int_{0}^{s} Y_{j}(l) d l-\beta s\right)} \varepsilon d s \\
& \leqslant \sup _{s \in[-\tau, N+\tau]}\left|e^{\beta s} x_{k}(s)\right|(N+\tau) \\
& \cdot \max _{s \in[-\tau, N+\tau]} e^{\left(\int_{0}^{s} \gamma_{j}(l) d l-\beta s\right)}+\varepsilon \frac{e^{\left(\int_{0}^{t} \gamma_{j}(s) d s-\beta t\right)}}{\gamma_{j}-\beta} .
\end{aligned}
$$

Combining (29) and (30) results in

$$
\begin{aligned}
Q_{3} & \leqslant e^{\beta \tau} e^{-\left(\int_{0}^{t} Y_{j}(s) d s-\beta t\right)} \bar{a}_{j} \sum_{k=1}^{n}\left|d_{j k}\right| G_{k} \\
& \cdot \int_{0}^{t} e^{\left(\int_{0}^{s} Y_{j}(l) d l-\beta s\right)}\left|e^{\beta\left(s-\tau_{j}(s)\right)} x_{k}\left(s-\tau_{j}(s)\right)\right| d s \\
& \leqslant\left(e^{\beta \tau} \bar{a}_{j} \sum_{k=1}^{n}\left|d_{j k}\right| G_{k} \sup _{s \in[-\tau, N+\tau]}\left|e^{\beta s} x_{k}(s)\right|(N+\tau)\right. \\
& \left.\max _{s \in[-\tau, N+\tau]} e^{\left(\int_{0}^{s} \Upsilon_{j}(l) d l-\beta s\right)}\right) e^{-\left(\int_{0}^{t} Y_{j}(s) d s-\beta t\right)} \\
& +\frac{e^{\beta \tau} \bar{a}_{j} \sum_{k=1}^{n}\left|d_{j k}\right| G_{k}}{\gamma_{j}-\beta} \varepsilon .
\end{aligned}
$$

Then the arbitrariness of $\varepsilon$ yields that $Q_{3} \rightarrow 0, t \rightarrow \infty$. 
It follows by (H3) and (25) that

$$
\begin{aligned}
Q_{4}= & \left|e^{\beta t} e^{-\int_{0}^{t} \Upsilon_{j}(s) d s} \sum_{0<t_{i}<t}\left(\rho_{j i}\left(x_{j}\left(t_{i}\right)\right)\right) e^{\int_{0}^{t_{i}} \Upsilon_{j}(s) d s}\right| \\
\leqslant & e^{\beta t} e^{-\int_{0}^{t} \Upsilon_{j}(s) d s} \sum_{0<t_{i}<t}\left|\rho_{j i}\left(x_{j}\left(t_{i}\right)\right)\right| e^{\int_{0}^{t_{i}} \Upsilon_{j}(s) d s} \\
\leqslant & e^{\beta t} e^{-\int_{0}^{t} \Upsilon_{j}(s) d s} \sum_{0<t_{i}<t} h_{j i}\left|x_{j}\left(t_{i}\right)\right| e^{\int_{0}^{t_{i}} \Upsilon_{j}(s) d s} \\
= & e^{-\left(\int_{0}^{t} \Upsilon_{j}(s) d s-\beta t\right)} \sum_{0<t_{i} \leqslant N} h_{j i}\left|x_{j}\left(t_{i}\right)\right| e^{\int_{0}^{t_{i}} \Upsilon_{j}(s) d s} \\
& +e^{\beta t} e^{-\int_{0}^{t} \Upsilon_{j}(s) d s} \sum_{N<t_{i}<t} h_{j i}\left|x_{j}\left(t_{i}\right)\right| e^{\int_{0}^{t_{i}} \Upsilon_{j}(s) d s} \\
= & e^{-\left(\int_{0}^{t} \Upsilon_{j}(s) d s-\beta t\right)} \sum_{0<t_{i} \leqslant N} h_{j i}\left|x_{j}\left(t_{i}\right)\right| e^{\int_{0}^{t_{i}} \Upsilon_{j}(s) d s} \\
& +e^{\beta t} e^{-\int_{0}^{t} \Upsilon_{j}(s) d s} \sum_{N<t_{i}<t} h_{j i}\left|x_{j}\left(t_{i}\right)\right| e^{\int_{0}^{t_{i}} \Upsilon_{j}(s) d s} .
\end{aligned}
$$

In addition,

$$
\frac{d}{d t}\left(\int_{0}^{t} \Upsilon_{j}(s) d s-\beta t\right)=\Upsilon_{j}(t)-\beta \geqslant \gamma-\beta>0,
$$

which implies the function $\left(\int_{0}^{t} \Upsilon_{j}(s) d s-\beta t\right)$ is an increasing function on $t \geqslant 0$. This yields

$$
\begin{aligned}
& e^{\beta t} e^{-\int_{0}^{t} Y_{j}(s) d s} \sum_{N<t_{i}<t} h_{j i}\left|x_{j}\left(t_{i}\right)\right| e^{\int_{0}^{t_{i}} \Upsilon_{j}(s) d s} \\
& =e^{\beta t} e^{-\int_{0}^{t} \Upsilon_{j}(s) d s} \sum_{N<t_{i} \leqslant t_{k}} h_{j i}\left(\left|x_{j}\left(t_{i}\right)\right| e^{\beta t_{i}}\right) e^{\left(\int_{0}^{t_{i}} \Upsilon_{j}(s) d s-\beta t_{i}\right)} \\
& \leqslant e^{\beta t} e^{-\int_{0}^{t} Y_{j}(s) d s} h_{j} \varepsilon \sum_{N<t_{i} \leqslant t_{k}} \mu e^{\left(\int_{0}^{t_{i}} \Upsilon_{j}(s) d s-\beta t_{i}\right)} \\
& \leqslant e^{\beta t} e^{-\int_{0}^{t} \Upsilon_{j}(s) d s} h_{j} \varepsilon\left[\sum_{N<t_{i}<t_{k}}\left(e^{\left(\int_{0}^{t_{i}} \Upsilon_{j}(s) d s-\beta t_{i}\right)}\left(t_{i+1}-t_{i}\right)\right)\right. \\
& \left.+\mu e^{\left(\int_{0}^{t_{k}} \Upsilon_{j}(s) d s-\beta t_{k}\right)}\right] \\
& \leqslant e^{\beta t} e^{-\int_{0}^{t} \Upsilon_{j}(s) d s} h_{j} \varepsilon\left[\int_{N}^{t} e^{\left(\int_{0}^{s} \Upsilon_{j}(l) d l-\beta s\right)} d s\right. \\
& \left.+\mu e^{\left(\int_{0}^{t} \Upsilon_{j}(s) d s-\beta t\right)}\right] \leqslant e^{\beta t} e^{-\int_{0}^{t} \Upsilon_{j}(s) d s} h_{j} \frac{\varepsilon}{\gamma-\beta} \\
& +\int_{N}^{t} e^{\left(\int_{0}^{s} \Upsilon_{j}(l) d l-\beta s\right)} d\left(\int_{0}^{s} \Upsilon_{j}(l) d l-\beta s\right)+\mu h_{j} \varepsilon \\
& \leqslant h_{j} \frac{\varepsilon^{2}}{\gamma-\beta}+\mu h_{j} \varepsilon .
\end{aligned}
$$

From (32) and (34), the arbitrariness of $\varepsilon$ yields that $Q_{4} \rightarrow$ $0, t \rightarrow \infty$.
So we can deduce from the above analysis that $\left|e^{\beta t} \Phi\left(x_{j}\right)\right| \rightarrow 0, t \rightarrow+\infty$, and hence the condition (c) is satisfied.

Next we are to prove that condition (d) holds in $\Phi\left(\Theta_{j}\right)$.

Indeed, for a given $t_{i}$, it follows from (20) that

$$
\Phi(x)\left(t_{i}+r\right)-\Phi(x)\left(t_{i}\right)=P_{1}+P_{2},
$$

where

$$
\begin{aligned}
P_{1} & =\left\{e^{-\int_{0}^{t_{i}+r} \Upsilon_{j}(s) d s} \phi_{j}(0)\right. \\
& +\int_{0}^{t_{i}+r} e^{-\int_{s}^{t_{i}+r} \Upsilon_{j}(l) d l}\left[a_{j}\left(x_{j}(s)\right)\right. \\
& \left.\left.\cdot \sum_{k=1}^{n}\left(c_{j k} f_{k}\left(x_{k}(s)\right)+d_{j k} g_{k}\left(x_{k}\left(s-\tau_{j}(s)\right)\right)\right)\right] d s\right\} \\
& -\left\{e^{-\int_{0}^{t_{i}} \Upsilon_{j}(s) d s} \phi_{j}(0)+\int_{0}^{t_{i}} e^{-\int_{s}^{t_{i}} \Upsilon_{j}(l) d l}\left[a_{j}\left(x_{j}(s)\right)\right.\right. \\
& \left.\left.\cdot \sum_{k=1}^{n}\left(c_{j k} f_{k}\left(x_{k}(s)\right)+d_{j k} g_{k}\left(x_{k}\left(s-\tau_{j}(s)\right)\right)\right)\right] d s\right\}, \\
P_{2} & =e^{-\int_{0}^{t_{i}+r} \Upsilon_{j}(s) d s} \sum_{0<t_{k}<t_{i}+r}\left(\rho_{j k}\left(x_{j}\left(t_{k}\right)\right)\right) e^{\int_{0}^{t_{k}} \Upsilon_{j}(s) d s} \\
& -e^{-\int_{0}^{t_{i}} \Upsilon_{j}(s) d s} \sum_{0<t_{k}<t_{i}}\left(\rho_{j k}\left(x_{j}\left(t_{k}\right)\right)\right) e^{\int_{0}^{t_{k}} \Upsilon_{j}(s) d s} .
\end{aligned}
$$

It is obvious that $P_{1} \rightarrow 0$ as $r \rightarrow 0$. From (36), we can get by letting $r<0$ be small enough

$$
\begin{aligned}
P_{2}= & \left(e^{-\int_{0}^{t_{i}+r} \Upsilon_{j}(s) d s}-e^{-\int_{0}^{t_{i}} \Upsilon_{j}(s) d s}\right) \\
& \cdot \sum_{0<t_{k}<t_{i}}\left(\rho_{j k}\left(x_{j}\left(t_{k}\right)\right)\right) e^{\int_{0}^{t_{k}} \Upsilon_{j}(s) d s},
\end{aligned}
$$

which implies that $\lim _{r \rightarrow 0^{-}} P_{2}=0$. On the other hand, letting $r>0$ be small enough, we have

$$
\begin{aligned}
P_{2} & =e^{-\int_{0}^{t_{i}+r} \Upsilon_{j}(s) d s}\left[\sum_{0<t_{k}<t_{i}}\left(\rho_{j k}\left(x_{j}\left(t_{k}\right)\right) e^{\int_{0}^{t_{k}} \Upsilon_{j}(s) d s}\right)\right. \\
& \left.+\rho_{j i}\left(x_{j}\left(t_{i}\right)\right) e^{\int_{0}^{t_{i}} \Upsilon_{j}(s) d s}\right] \\
& -e^{-\int_{0}^{t_{i}} \Upsilon_{j}(s) d s} \sum_{0<t_{k}<t_{i}}\left(\rho_{j k}\left(x_{j}\left(t_{k}\right)\right)\right) e^{\int_{0}^{t_{k}} \Upsilon_{j}(s) d s} \\
& =\left(e^{-\int_{0}^{t_{i}+r} \Upsilon_{j}(s) d s}-e^{-\int_{0}^{t_{i}} \Upsilon_{j}(s) d s}\right) \\
& \cdot \sum_{0<t_{k}<t_{i}}\left(\rho_{j k}\left(x_{j}\left(t_{k}\right)\right) e^{\int_{0}^{t_{k}} \Upsilon_{j}(s) d s}\right) \\
& +e^{-\int_{0}^{t_{i}+r} \Upsilon_{j}(s) d s} \rho_{j i}\left(x_{j}\left(t_{i}\right)\right) e^{\int_{0}^{t_{i}} \Upsilon_{j}(s) d s},
\end{aligned}
$$


which leads to $\lim _{r \rightarrow 0^{+}} P_{2}=\rho_{j i}\left(x_{j}\left(t_{i}\right)\right)$. Hence, condition (d) holds in $\Phi\left(\Theta_{j}\right)$.

It is derived by the above analysis that $\Phi\left(\Theta_{j}\right) \subset \Theta_{j}$ for all $j$.

Step 3. We claim that $\Phi$ is a contraction mapping on $\Theta$.

Indeed, for $x(t)=\left(x_{1}(t), \ldots, x_{j}(t), \ldots, x_{n}(t)\right)^{T} \in \Theta$ and $y(t)=\left(y_{1}(t), \ldots, y_{j}(t), \ldots, y_{n}(t)\right)^{T} \in \Theta$, we have

$$
\left|\Phi\left(x_{j}\right)(t)-\Phi\left(y_{j}\right)(t)\right| \leqslant J_{j 1}+J_{j 2}+J_{j 3},
$$

where

$$
\begin{aligned}
J_{j 1} & =\int_{0}^{t} e^{-\int_{s}^{t} Y_{j}(l) d l} \mid a_{j}\left(x_{j}(s)\right) \sum_{k=1}^{n} c_{j k} f_{k}\left(x_{k}(s)\right) \\
& -a_{j}\left(y_{j}(s)\right) \sum_{k=1}^{n} c_{j k} f_{k}\left(y_{k}(s)\right) \mid d s
\end{aligned}
$$

$$
\begin{aligned}
J_{j 2} & =\int_{0}^{t} e^{-\int_{s}^{t} \Upsilon_{j}(l) d l} \mid a_{j}\left(x_{j}(s)\right) \\
& \cdot \sum_{k=1}^{n} d_{j k} g_{k}\left(x_{k}\left(s-\tau_{j}(s)\right)\right)-a_{j}\left(y_{j}(s)\right) \\
& \cdot \sum_{k=1}^{n} d_{j k} g_{k}\left(y_{k}\left(s-\tau_{j}(s)\right)\right) \mid d s, \\
J_{j 3} & =e^{-\int_{0}^{t} \Upsilon_{j}(s) d s} \sum_{0<t_{i}<t}\left(\left|\rho_{j i}\left(x_{j}\left(t_{i}\right)\right)-\rho_{j i}\left(y_{j}\left(t_{i}\right)\right)\right|\right.
\end{aligned}
$$$$
\left.\cdot e^{\int_{0}^{t_{i}} \Upsilon_{j}(s) d s}\right) \cdot
$$

It follows by the triangle inequality, $(\mathrm{H} 1),(\mathrm{H} 2)$, and the differential mean value theorem that

$$
\begin{aligned}
& \left|a_{j}\left(x_{j}(s)\right) \sum_{k=1}^{n} c_{j k} f_{k}\left(x_{k}(s)\right)-a_{j}\left(y_{j}(s)\right) \sum_{k=1}^{n} c_{j k} f_{k}\left(y_{k}(s)\right)\right| \leqslant \sum_{k=1}^{n}\left[| c _ { j k } | \left(\left|a_{j}\left(x_{j}(s)\right) f_{k}\left(x_{k}(s)\right)-a_{j}\left(x_{j}(s)\right) f_{k}\left(y_{k}(s)\right)\right|\right.\right. \\
& \left.\left.\quad+\left|a_{j}\left(x_{j}(s)\right) f_{k}\left(y_{k}(s)\right)-a_{j}\left(y_{j}(s)\right) f_{k}\left(y_{k}(s)\right)\right|\right)\right] \leqslant \sum_{k=1}^{n}\left(\left|c_{j k}\right| \bar{a}_{j} F_{k}\left|x_{k}(s)-y_{k}(s)\right|\right)+\left(\sum_{k=1}^{n}\left|c_{j k}\right| \bar{F}_{k}\right) \tilde{a}_{j} \mid x_{j}(s) \\
& \quad-y_{j}(s) \mid, \\
& \quad \cdot\left|a_{j}\left(x_{j}(s)\right) \sum_{k=1}^{n} d_{j k} g_{k}\left(x_{k}\left(s-\tau_{j}(s)\right)\right)-a_{j}\left(y_{j}(s)\right) \sum_{k=1}^{n} d_{j k} g_{k}\left(y_{k}\left(s-\tau_{j}(s)\right)\right)\right| \leqslant \sum_{k=1}^{n}\left[\left|d_{j k}\right|\right. \\
& \left.\quad+\mid a_{j}\left(x_{j}(s)\right) g_{k}\left(y_{k}\left(s-\tau_{j}(s)\right)\right)-a_{j}\left(x_{j}(s)\right)\right)-a_{k}\left(y_{k}\left(s-\tau_{j}(s)\right)\right) \mid \\
& \left.\quad-y_{k}\left(s-\tau_{j}(s)\right) \mid\right)+\left(\sum_{k=1}^{n}\left|d_{j k}\right| \bar{G}_{k}\right) \tilde{a}_{j}\left|x_{j}(s)-y_{j}(s)\right| .
\end{aligned}
$$

On the other hand,

$$
\begin{aligned}
\int_{0}^{t} e^{\int_{0}^{s} Y_{j}(l) d l} d s & =\int_{0}^{t} \frac{e^{\int_{0}^{s} \Upsilon_{j}(l) d l}}{\Upsilon_{j}(s)} d\left(\int_{0}^{s} \Upsilon_{j}(l) d l\right) \\
& \leqslant \int_{0}^{t} \frac{e^{\int_{0}^{s} \Upsilon_{j}(l) d l}}{\gamma_{j}} d\left(\int_{0}^{s} \Upsilon_{j}(l) d l\right)
\end{aligned}
$$

$$
\leqslant \frac{e^{\int_{0}^{t} \gamma_{j}(s) d s}}{\gamma_{j}}
$$

$$
\begin{aligned}
J_{j 1} & \leqslant \int_{0}^{t} e^{-\int_{s}^{t} Y_{j}(l) d l}\left[\sum_{k=1}^{n}\left(\left|c_{j k}\right| \bar{a}_{j} F_{k}\left|x_{k}(s)-y_{k}(s)\right|\right)+\left(\sum_{k=1}^{n}\left|c_{j k}\right| \bar{F}_{k}\right) \widetilde{a}_{j}\left|x_{j}(s)-y_{j}(s)\right|\right] d s \\
& \leqslant e^{-\int_{0}^{t} Y_{j}(s) d s}\left[\sum_{k=1}^{n}\left(\left|c_{j k}\right| \bar{a}_{j} F_{k} \sup _{s \geqslant-\tau}\left|x_{k}(s)-y_{k}(s)\right|\right)+\left(\sum_{k=1}^{n}\left|c_{j k}\right| \bar{F}_{k}\right) \widetilde{a}_{j} \sup _{s \geqslant-\tau}\left|x_{j}(s)-y_{j}(s)\right|\right] \int_{0}^{t} e^{\int_{0}^{s} Y_{j}(l) d l} d s
\end{aligned}
$$




$$
\begin{aligned}
& \leqslant \frac{\left(\max _{k \in \mathcal{N}}\left|c_{j k}\right| F_{k}\right) \bar{a}_{j}}{\gamma_{j}} \operatorname{dist}(x, y)+\frac{\left(\sum_{k=1}^{n}\left|c_{j k}\right| \bar{F}_{k}\right) \tilde{a}_{j}}{\gamma_{j}} \sup _{t \geqslant-\tau}\left|x_{j}(t)-y_{j}(t)\right|, \\
& J_{j 2} \leqslant \int_{0}^{t} e^{-\int_{s}^{t} \gamma_{j}(l) d l}\left[\sum_{k=1}^{n}\left(\left|d_{j k}\right| \bar{a}_{j} G_{k}\left|x_{k}\left(s-\tau_{j}(s)\right)-y_{k}\left(s-\tau_{j}(s)\right)\right|\right)+\left(\sum_{k=1}^{n}\left|d_{j k}\right| \bar{G}_{k}\right) \widetilde{a}_{j}\left|x_{j}(s)-y_{j}(s)\right|\right] d s \\
& \leqslant e^{-\int_{0}^{t} Y_{j}(s) d s}\left[\sum_{k=1}^{n}\left(\left|d_{j k}\right| \bar{a}_{j} G_{k} \sup _{s \geqslant-\tau}\left|x_{k}(s)-y_{k}(s)\right|\right)+\left(\sum_{k=1}^{n}\left|d_{j k}\right| \bar{G}_{k}\right) \widetilde{a}_{j} \sup _{s \geqslant-\tau}\left|x_{j}(s)-y_{j}(s)\right|\right] \int_{0}^{t} e^{\int_{0}^{s} Y_{j}(l) d l} d s \\
& \leqslant \frac{\max _{k \in \mathcal{N}}\left(\left|d_{j k}\right| G_{k}\right) \bar{a}_{j}}{\gamma_{j}} \operatorname{dist}(x, y)+\frac{\left(\sum_{k=1}^{n}\left|d_{j k}\right| \bar{G}_{k}\right) \tilde{a}_{j}}{\gamma_{j}} \sup _{t \geqslant-\tau}\left|x_{j}(t)-y_{j}(t)\right| \text {, } \\
& J_{j 3} \leqslant e^{-\int_{0}^{t} Y_{j}(s) d s} \sum_{0<t_{i}<t}\left(h_{j i}\left|x_{j}\left(t_{i}\right)-y_{j}\left(t_{i}\right)\right| e^{\int_{0}^{t_{i}} Y_{j}(s) d s}\right) \leqslant h_{j} e^{-\int_{0}^{t} Y_{j}(s) d s} \sup _{t \geqslant-\tau}\left|x_{j}\left(t_{i}\right)-y_{j}\left(t_{i}\right)\right| \sum_{0<t_{i}<t}\left(\mu e^{\int_{0}^{t_{i}} Y_{j}(s) d s}\right) \\
& \leqslant h_{j} e^{-\int_{0}^{t} Y_{j}(s) d s} \sup _{t \geqslant-\tau}\left|x_{j}\left(t_{i}\right)-y_{j}\left(t_{i}\right)\right|\left(\sum_{0<t_{i}<t_{k}} e^{\int_{0}^{t_{j}} Y_{j}(s) d s}\left(t_{r+1}-t_{r}\right)+\mu e^{\int_{0}^{t_{k}} Y_{j}(s) d s}\right) \leqslant h_{j} e^{-\int_{0}^{t} Y_{j}(s) d s} \sup _{t \geqslant-\tau}\left|x_{j}\left(t_{i}\right)-y_{j}\left(t_{i}\right)\right| \\
& \cdot\left(\int_{0}^{t} e^{\int_{0}^{s} Y_{j}(l) d l} d s+\mu e^{\int_{0}^{t} Y_{j}(s) d s}\right) \leqslant h_{j}\left(\frac{1}{\gamma_{j}}+\mu\right) \sup _{t \geqslant-\tau}\left|x_{j}\left(t_{i}\right)-y_{j}\left(t_{i}\right)\right| .
\end{aligned}
$$

So we have

$$
\begin{aligned}
& \sup _{t \geqslant-\tau}\left|\Phi\left(x_{j}\right)(t)-\Phi\left(y_{j}\right)(t)\right| \leqslant \sup _{t \geqslant-\tau}\left(J_{j 1}+J_{j 2}+J_{j 3}\right) \\
& \leqslant \frac{\left(\max _{k \in \mathcal{N}}\left|c_{j k}\right| F_{k}\right) \bar{a}_{j}}{\gamma_{j}} \operatorname{dist}(x, y) \\
& +\frac{\left(\sum_{k=1}^{n}\left|c_{j k}\right| \bar{F}_{k}\right) \tilde{a}_{j}}{\gamma_{j}} \sup _{t \geqslant-\tau}\left|x_{j}(t)-y_{j}(t)\right| \\
& +\frac{\max _{k \in \mathcal{N}}\left(\left|d_{j k}\right| G_{k}\right) \bar{a}_{j}}{\gamma_{j}} \operatorname{dist}(x, y) \\
& +\frac{\left(\sum_{k=1}^{n}\left|d_{j k}\right| \bar{G}_{k}\right) \tilde{a}_{j}}{\gamma_{j}} \sup _{t \geqslant-\tau}\left|x_{j}(t)-y_{j}(t)\right| \\
& +h_{j}\left(\frac{1}{\gamma_{j}}+\mu\right) \sup _{t \geqslant-\tau}\left|x_{j}\left(t_{i}\right)-y_{j}\left(t_{i}\right)\right| \\
& \leqslant \frac{\left(\max _{k \in \mathcal{N}}\left|c_{j k}\right| F_{k}\right) \bar{a}_{j}+\max _{k \in \mathcal{N}}\left(\left|d_{j k}\right| G_{k}\right) \bar{a}_{j}}{\gamma_{j}} \\
& \cdot \operatorname{dist}(x, y) \\
& +\frac{\left(\sum_{k=1}^{n}\left|c_{j k}\right| \bar{F}_{k}\right) \tilde{a}_{j}+\left(\sum_{k=1}^{n}\left|d_{j k}\right| \bar{G}_{k}\right) \tilde{a}_{j}+h_{j}+\mu \gamma_{j}}{\gamma_{j}} \\
& \cdot \sup _{t \geqslant-\tau}\left|x_{j}(t)-y_{j}(t)\right|
\end{aligned}
$$

Therefore, we can derive by the above analysis and the LMI condition

$$
\begin{aligned}
& \operatorname{dist}(\Phi(x), \Phi(y))=\sum_{j=1}^{n} \sup _{t \geqslant-\tau}\left|\Phi\left(x_{j}\right)(t)-\Phi\left(y_{j}\right)(t)\right| \\
& \leqslant \sum_{j=1}^{n}\left\{\frac{\left(\max _{k \in \mathcal{N}}\left|c_{j k}\right| F_{k}\right) \bar{a}_{j}+\max _{k \in \mathcal{N}}\left(\left|d_{j k}\right| G_{k}\right) \bar{a}_{j}}{\gamma_{j}}\right. \\
& \cdot \operatorname{dist}(x, y) \\
& +\frac{\left(\sum_{k=1}^{n}\left|c_{j k}\right| \bar{F}_{k}\right) \tilde{a}_{j}+\left(\sum_{k=1}^{n}\left|d_{j k}\right| \bar{G}_{k}\right) \tilde{a}_{j}+h_{j}+\mu \gamma_{j}}{\gamma_{j}} \\
& \left.\cdot \sup _{t \geqslant-\tau}\left|x_{j}(t)-y_{j}(t)\right|\right\} \leqslant \alpha \operatorname{dist}(x, y),
\end{aligned}
$$

which implies that $\Phi$ is a contraction mapping on $\Theta$. And then the contraction mapping theorem yields the idea that $\Phi$ has the fixed point $x(t)$ on $\Theta$, which implies that $x(t)$ is the solution for system (1), satisfying $e^{\beta t}\|x(t)\| \rightarrow 0$ as $t \rightarrow \infty$. And then the proof is completed.

Remark 6. As far as we can know, there is not any previous literature related to the fixed point theory where LMI-based stability criteria were presented, except for [15]. In this paper, the LMI-based stability criterion is the first time to be proposed for impulsive CGNNs via fixed point theorems. This is another main contribution of this paper. 


\section{Numerical Example}

Example 1. Consider

$$
\begin{aligned}
& \frac{d x_{1}(t)}{d t}=-a_{1}\left(x_{1}(t)\right)\left\{b_{1}\left(x_{1}(t)\right)\right. \\
& \left.-\sum_{k=1}^{2}\left[c_{1 k} f_{k}\left(x_{k}(t)\right)+d_{1 k} g_{k}\left(x_{k}\left(t-\tau_{1}(t)\right)\right)\right]\right\}, \\
& t \geqslant 0, t \neq t_{i}, \\
& \frac{d x_{2}(t)}{d t}=-a_{2}\left(x_{2}(t)\right)\left\{b_{2}\left(x_{2}(t)\right)\right. \\
& \left.-\sum_{k=1}^{2}\left[c_{2 k} f_{k}\left(x_{k}(t)\right)+d_{2 k} g_{k}\left(x_{k}\left(t-\tau_{2}(t)\right)\right)\right]\right\}, \\
& \Delta x_{j}\left(t_{i}\right)=x_{j}\left(t_{i}^{+}\right)-x_{j}\left(t_{i}\right)=\rho_{j i}\left(x_{j}\left(t_{i}\right)\right), \\
& x_{j}(\theta)=\phi_{j}(\theta), \quad-\tau \leqslant \theta \leqslant 0, \quad j \in \mathcal{N}, \quad t \neq t_{i},
\end{aligned}
$$

where $j \in \mathcal{N} \triangleq\{1,2\}, b_{1}\left(x_{1}(t)\right)=2 x_{1}(t), b_{2}\left(x_{2}(t)\right)=2 x_{2}(t)$, $a_{1}\left(x_{1}(t)\right)=a_{2}\left(x_{2}(t)\right)=2+\cos t$, and then $a_{j}(r) b_{j}(r)=2(2+$ $\cos t) r=\Upsilon_{j}(t) r$; hence $\Upsilon_{j}(t)=2(2+\cos t)$. Let $\gamma_{j}=2$, and then $\Upsilon_{j}(t)=2(2+\cos t) \geqslant \gamma_{j}>0$, let $\bar{a}_{j}=3$; then $0<$ $a_{j}(r)=(2+\cos t) \cdot r^{0} \leqslant \bar{a}_{j}$, and $a_{j}^{\prime}(r)=0$. So we let $\widetilde{a}_{j}=0$, and $\left|a_{j}^{\prime}(r)\right| \leqslant \tilde{a}_{j}$. Let $\int_{0}^{t} \Upsilon_{j}(s) d s=4 t+2 \sin t$. Let $\beta=1>0$, and then $\liminf \operatorname{in}_{t \rightarrow+\infty}\left(\int_{0}^{t} \Upsilon_{j}(s) d s / t\right)=4>\beta$ and $\gamma_{j}>\beta$. Let $f_{i}(r)=\sin ^{2}(r / 10)=g_{i}(r), i=1,2$; then $\bar{F}_{i}=\bar{G}_{i}=1$, $b_{i}(0)=f_{i}(0)=g_{i}(0)=0$, and $F_{i}=G_{i}=0.2, i=1,2$. Define $\rho_{j i}=\arctan \left(1.7 x_{j}\left(t_{i}\right)\right)$ and $t_{i}-t_{i-1}=0.5 i$ for $j=$ $1,2, i=1,2, \ldots$. Let $h_{j i}=1.7, h_{j}=3.4$, and $\mu=0.5$, and then $h_{j i} \leqslant h_{j} \mu$ holds.

In addition, let $c_{j k}=d_{j k}=0.05$ for $j, k \in \mathcal{N}$; then we can use Matlab LMI toolbox to solve the LMI condition in Theorem 4 and obtain

$$
\alpha=0.8351<1 \text {. }
$$

According to Theorem 4, system (46) is globally exponentially stable.

Remark 7. To compare the upper bounds of time delay and impulse in various related literature, we need to compute and compare the ratios between the maximum of allowable impulse and maximum of parameters in various related literature, because the maximum of allowable impulse may rise as parameters of numerical examples become bigger. From Table 1, the ratios of Example 1 (ours) are bigger than those of $[1,4,10,11]$ to some extent. In addition, impulse value of [9, Example 4.1] is less than 1 while ours of Example 1 is $1.7>1$. It is well known that bigger impulse gives
TABLE 1: Ratios between allowable upper bounds of impulse $\mathscr{C}$ and maximum of parameters $\mathscr{P}$.

\begin{tabular}{lccc}
\hline & $\mathscr{C}$ & $\mathscr{P}$ & $\mathscr{C} / \mathscr{P}$ \\
\hline Example 1 (ours) & 1.7 & 2 & 0.850 \\
[1, Example 1] & 1 & 3 & 0.333 \\
[4, Example 1] & 0.8 & 3.5 & 0.2286 \\
[9, Example 4.1] & 0.55 & 0.2 & 2.75 \\
[10, Example Case 1] & 0.81 & 4.8 & 0.1688 \\
[11, Example] & 0.7 & 6 & 0.1167 \\
\hline
\end{tabular}

bigger influence on the stability. Thereby, the large allowable variation range of impulse illustrates the effectiveness of our new stability criterion.

Remark 8. The super limit $\tau$ of time delays is actually infinite.

Remark 9. As far as we know, it is the first time to use the contraction mapping theorem to prove the stability for CGNNs. The method employed in this paper is different from those of related literature $[20,21]$.

Remark 10. Of course, [1, 4, 9-11] involved more complicated systems than ours, such as reaction-diffusion phenomena, stochastic Markovian jumping, and parameters uncertainty. Moreover, fixed point theories may not be employed to such complicated systems. Thereby, we can not claim that the new stability criterion is better than the criteria of $[1,4,9-11]$ in all aspects, for those results obtained in [1, 4, 9-11] solved what Theorem 4 (ours) cannot involve. However, a further research on applications of fixed point theories may have the widest appeal of all. This is another purpose of writing this paper.

\section{Conflict of Interests}

The authors declare that there is no conflict of interests regarding the publication of this paper.

\section{Acknowledgments}

This work was supported by Scientific Research Fund of Science Technology Department of Sichuan Province (2012JY010) and by Sichuan Educational Committee Science Foundation (08ZB002, 12ZB349, and 14ZA0274).

\section{References}

[1] K. Li and Q. Song, "Exponential stability of impulsive CohenGrossberg neural networks with time-varying delays and reaction-diffusion terms," Neurocomputing, vol. 72, no. 1-3, pp. 231-240, 2008.

[2] R. Rao and S. Zhong, "Existence of exponential p-stability nonconstant equilibrium of markovian jumping nonlinear diffusion equations via ekeland variational principle," Advances in Mathematical Physics, vol. 2015, Article ID 812150, 10 pages, 2015. 
[3] J. Tian, W. Xiong, and F. Xu, "Improved delay-partitioning method to stability analysis for neural networks with discrete and distributed time-varying delays," Applied Mathematics and Computation, vol. 233, pp. 152-164, 2014.

[4] Y. Zhang and Q. Luo, "Global exponential stability of impulsive delayed reaction-diffusion neural networks via Hardy-Poincarè inequality," Neurocomputing, vol. 83, no. 15, pp. 198-204, 2012.

[5] X. Liu, S. Zhong, and X. Ding, "Robust exponential stability of impulsive switched systems with switching delays: a Razumikhin approach," Communications in Nonlinear Science and Numerical Simulation, vol. 17, no. 4, pp. 1805-1812, 2012.

[6] J. Cao and Q. Song, "Stability in Cohen-Grossberg-type bidirectional associative memory neural networks with time-varying delays," Nonlinearity, vol. 19, no. 7, pp. 1601-1617, 2006.

[7] J. Cao and X. Li, "Stability in delayed Cohen-Grossberg neural networks. LMI optimization approach," Physica D: Nonlinear Phenomena, vol. 212, no. 1-2, pp. 54-65, 2005.

[8] K. Yuan and J. Cao, "An analysis of global asymptotic stability of delayed Cohen-Grossberg neural networks via nonsmooth analysis," IEEE Transactions on Circuits and Systems I: Regular Papers, vol. 52, no. 9, pp. 1854-1861, 2005.

[9] R. Rao, "Delay-dependent exponential stability for nonlinear reaction-diffusion uncertain Cohen-Grossberg neural networks with partially known transition rates via Hardy-Poincaré inequality," Chinese Annals of Mathematics B, vol. 35, no. 4, pp. 575-598, 2014.

[10] R. Rao, S. Zhong, and X. Wang, "Stochastic stability criteria with LMI conditions for Markovian jumping impulsive BAM neural networks with mode-dependent time-varying delays and nonlinear reaction-diffusion," Communications in Nonlinear Science and Numerical Simulation, vol. 19, no. 1, pp. 258-273, 2014.

[11] J. Pan, X. Liu, and S. Zhong, "Stability criteria for impulsive reaction-diffusion Cohen-Grossberg neural networks with time-varying delays," Mathematical and Computer Modelling, vol. 51, no. 9-10, pp. 1037-1050, 2010.

[12] R. Rao, X. Wang, and S. Zhong, "LMI-based stability criterion for impulsive delays markovian jumping time-delays reaction-diffusion BAM neural networks via gronwall-bellmantype impulsive integral inequality," Mathematical Problems in Engineering, vol. 2015, Article ID 185854, 11 pages, 2015.

[13] T. A. Burton, "Liapunov functionals, fixed points, and stability by Krasnoselskiis theorem," Nonlinear Studies, vol. 9, no. 2, pp. 181-190, 2002.

[14] T. A. Burton, "Fixed points and stability of a nonconvolution equation," Proceedings of the American Mathematical Society, vol. 132, no. 12, pp. 3679-3687, 2004.

[15] R. Rao and Z. Pu, "LMI-based stability criterion of impulsive TS fuzzy dynamic equations via fixed point theory," Abstract and Applied Analysis, vol. 2013, Article ID 261353, 9 pages, 2013.

[16] S.-M. Jung, "A fixed point approach to the stability of a Volterra integral equation," Fixed Point Theory and Applications, vol. 2007, Article ID 57064, 9 pages, 2007.

[17] J. Luo, "Fixed points and stability of neutral stochastic delay differential equations," Journal of Mathematical Analysis and Applications, vol. 334, no. 1, pp. 431-440, 2007.

[18] B. Zhang, "Fixed points and stability in differential equations with variable delays," Nonlinear Analysis: Theory, Methods \& Applications, vol. 63, no. 5-7, pp. e233-e242, 2005.

[19] M. Wu, N.-J. Huang, and C.-W. Zhao, "Fixed points and stability in neutral stochastic differential equations with variable delays,"
Fixed Point Theory and Applications, vol. 2008, Article ID 407352, 11 pages, 2008.

[20] X. Yang, C. Huang, D. Zhang, and Y. Long, "Dynamics of cohengrossberg neural networks with mixed delays and impulses," Abstract and Applied Analysis, vol. 2008, Article ID 432341, 14 pages, 2008.

[21] H. Zhu and C. Feng, "Existence and global uniform asymptotic stability of pseudo almost periodic solutions for CohenGrossberg neural networks with discrete and distributed delays," Mathematical Problems in Engineering, vol. 2014, Article ID 968404, 10 pages, 2014.

[22] O. Hadzic and E. Pap, Fixed Point Theory in Probabilistic Metric Spaces, Springer, Dordrecht, The Netherlands, 2001. 


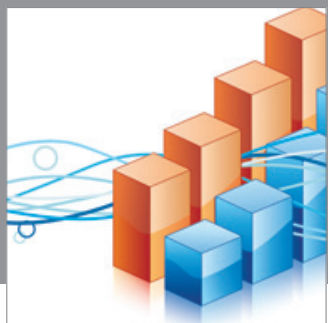

Advances in

Operations Research

mansans

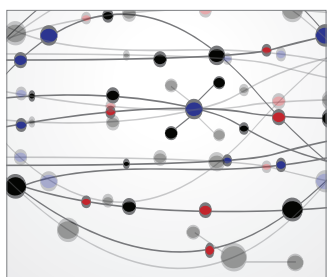

The Scientific World Journal
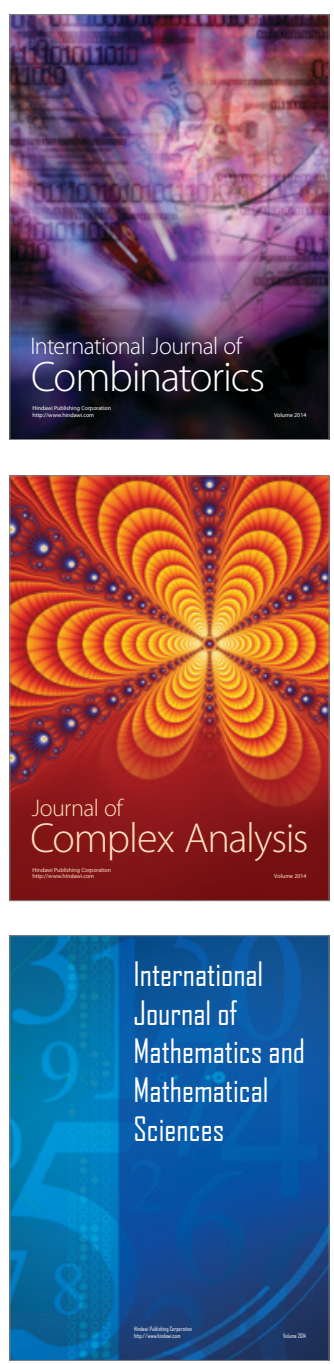
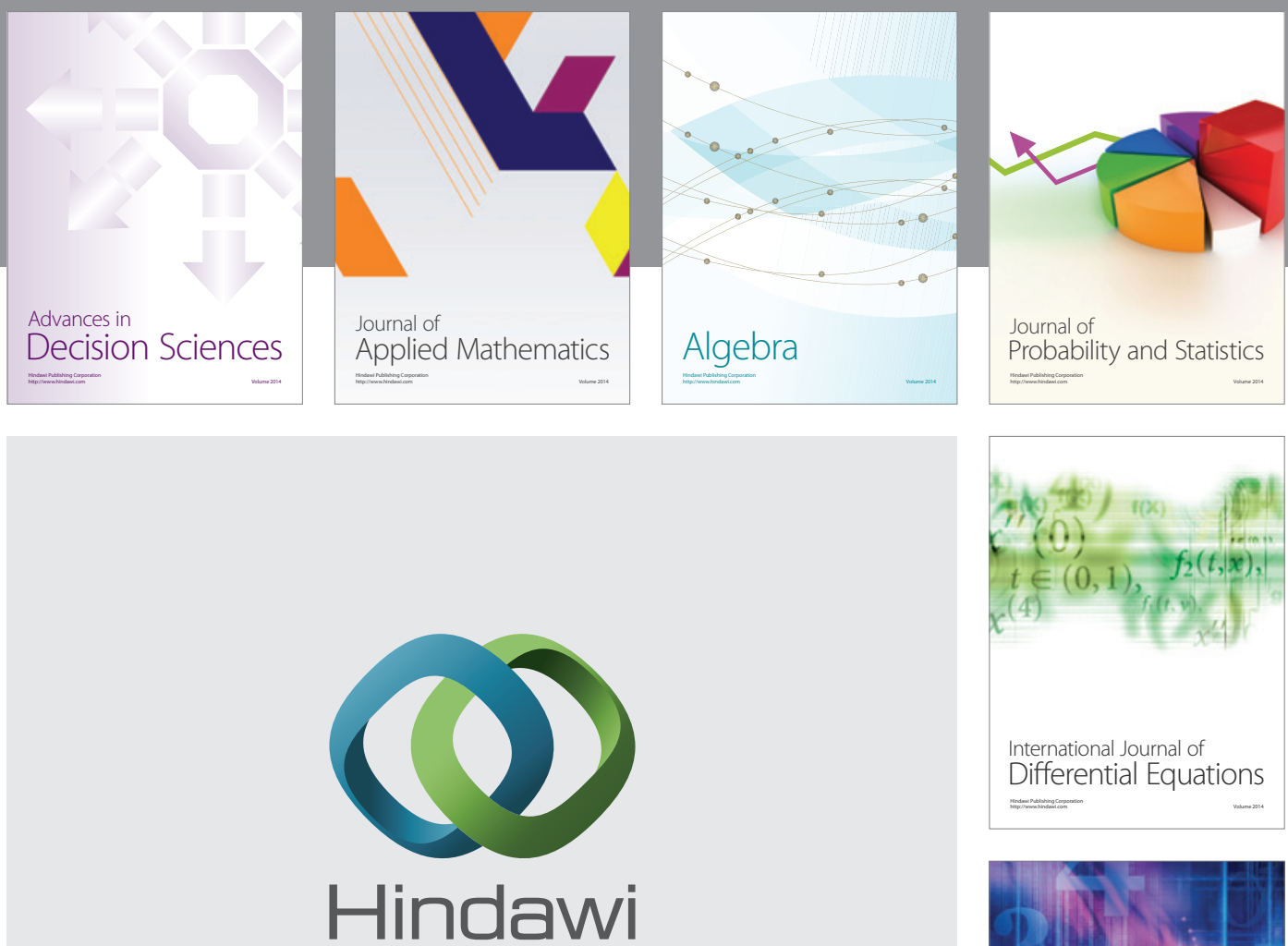

Submit your manuscripts at http://www.hindawi.com
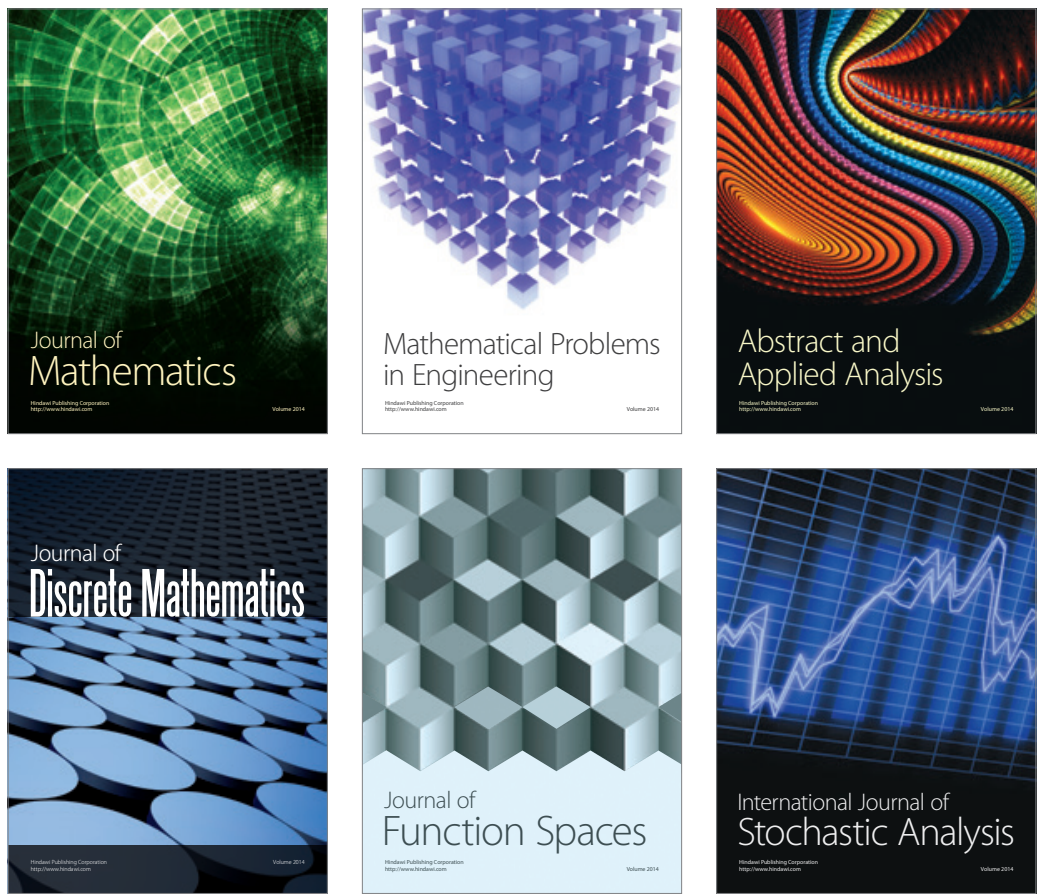

Journal of

Function Spaces

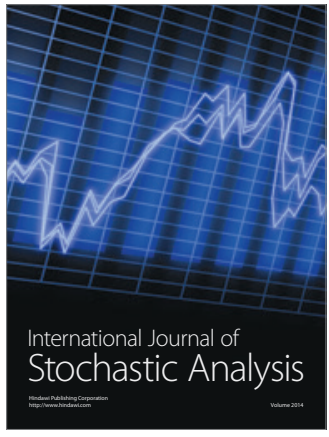

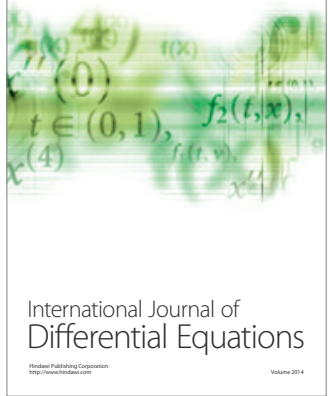
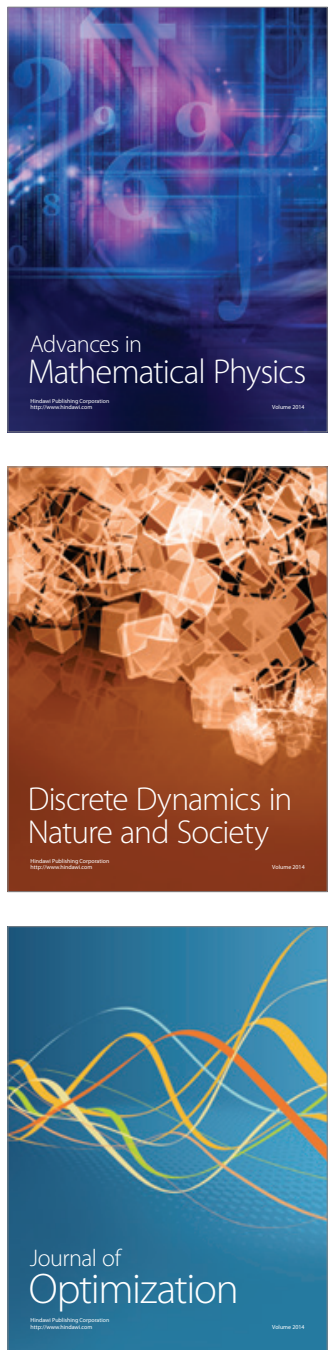